\title{
Racial disparity in long-term mortality rate after hospitalization for myocardial infarction: The Atherosclerosis Risk in Communities study
}

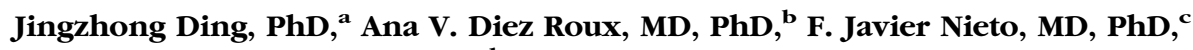
Robert L. McNamara, MD, MHS, ${ }^{\mathrm{d}}$ Jacqueline B. Hetmanski, MS, ${ }^{\mathrm{a}}$ Herman A. Taylor, Jr, MD, ${ }^{\mathrm{e}}$ and Herman A. Tyroler, MD ${ }^{\mathbf{f}}$ Baltimore, Md, New York, NY, Madison, Wis, Chinle, Ariz, Jackson, Miss, and Chapel Hill, NC

Background The underlying reasons why African American patients have a significantly higher mortality rate than European American patients after a myocardial infarction (MI) remain unclear. This study examined the racial disparity in mortality rates after $\mathrm{Ml}$ and possible explanatory factors.

Methods A prospective analysis was conducted within the Atherosclerosis Risk in Communities (ARIC) study, a community-based study of 15,792 middle-aged adults. From 1987 to 1998, 642 patients (471 European American and 171 African American) hospitalized for Ml without prior history of Ml were identified. Of these 642 patients, 129 (82 European American and 47 African American) died during follow-up.

Results Cox proportional hazard models were used to analyze the racial difference in mortality rate after MI. After adjusting for age and sex, the relative hazard (RH) comparing African American patients to European American patients was $1.80(95 \% \mathrm{Cl}, 1.24-2.61)$. The $\mathrm{RH}$ decreased after adjusting for vascular risk factors $(1.29 ; 95 \% \mathrm{Cl}, 0.83-2.00)$, socioeconomic position $(1.31 ; 95 \% \mathrm{Cl}, 0.83-2.09)$, severity of $\mathrm{Ml}(1.60 ; 95 \% \mathrm{Cl}, 1.05-2.45)$, and treatment $(1.36 ; 95 \%$ $\mathrm{Cl}, 0.92-2.00)$. In the final model, which included all factors aforementioned, the $\mathrm{RH}$ for race was $1.00(95 \% \mathrm{Cl}, 0.56$. 1.77).

Conclusions Our findings suggested that vascular risk factors, socioeconomic position, and treatment play major roles in the racial disparity in mortality rate after MI. (Am Heart J 2003;146:459-64.)

It has been observed that African American patients have a significantly higher mortality rate after myocardial infarction (MI) than European American patients. ${ }^{1,2}$ Predisposing vascular risk factors, such as hypertension and diabetes mellitus, have been suggested to contribute to the disparity. ${ }^{2,3}$ Socioeconomic position may play an important role in this adverse out-

From the Department of Epidemiology, Bloomberg School of Public Health, Johns Hop kins University, Baltimore, Md, Division of General Medicine, Columbia College of Physicians and Surgeons and Department of Epidemiology, Mailman School of Public Health, Columbia University, New York, NY, Department of Population Health Sciences, University of Wisconsin Medical School, Madison, Wis, Department of Internal Medicine, Chinle Comprehensive Healthcare Facility, Chinle, Ariz, Department of Medicine, University of Mississippi Medical Center, Jackson, Miss, Department of Epidemiology, School of Public Health, University of North Carolina, Chapel Hill, NC. The ARIC Study is carried out as a collaborative study supported by contracts from the National Heart, Lung, and Blood Institute (NO1-HC-55015, NO1-HC-55016, NO1-HC 55018, N01-HC-55019, N01-HC-55020, NO1-HC-55021, and N01-HC-55022).

Submitted July 29, 2002; accepted February 5, 2003.

Reprint requests: Jingzhong Ding, Department of Epidemiology, Bloomberg School of Public Health, Johns Hopkins University, 615 N Wolfe St, Room W6009, Baltimore, MD 21205.

E-mail: jding@jhsph.edu

(C) 2003, Mosby, Inc. All rights reserved.

$0002-8703 / 2003 / \$ 30.00+0$

doi:10.1016/S0002-8703/03/00228-X come in African American patients. ${ }^{4}$ Racial variation in treatment has also been suggested as a contributor to the disparity. ${ }^{5,6}$ However, most epidemiologic studies are hospital based and may involve selection bias. Long-term prospective studies are lacking. Moreover, information on severity of MI, treatment, time to access medical care, and other risk factors are usually not complete or well characterized. There are still controversies about the reasons for the racial disparity in prognosis after MI and the relative contributions of these factors.

A previous Atherosclerosis Risk in Communities (ARIC) study found that age-adjusted 28-day case fatalities of acute coronary heart disease were higher in African American patients than European American patients. ${ }^{7}$ However, the underlying mechanisms of the racial disparity remain unexplored. This study is a long-term, community-based study investigating mechanisms of the racial disparity in mortality rate after MI.

\section{Methods}

This study population is from the ARIC cohort, a prospective study conducted in 4 US communities: Forsyth County, 
NC; Jackson, Miss; suburban Minneapolis, Minn; and Washington County, Md. A total of 15,792 persons who were not institutionalized, aged 45 to 64 years at initial recruitment (1987-1989), were selected with probability sampling from each community. The response rate was $60 \%$. Since 1987 , annual follow-up interviews and clinical examinations were performed every 3 years. By the end of 1998, only 245 participants were lost to follow-up, leaving a follow-up proportion of $98.4 \%$. Informed consent was obtained from every participant. The ARIC study has been approved by the institutional review board of each participating institution. Further details of the ARIC Study can be found at the website http://www.bios.unc.edu/cscc/.

MI incidence was defined as the first hospitalization for a MI event from the baseline (1987-89) to December 31, 1998, in participants without previous MI history. Hospital admissions were identified initially through a review of hospital discharge indexes in the study area or review of death certificates or information elicited during the annual follow-up interview. Hospital chart abstraction was carried out for all the hospitalizations with these International Classification of Diseases 9th revision-Clinical Modification codes for primary or secondary discharge diagnosis: 402,410 to $414,427,428$, and 518.4. A review of death certificates or annual follow-up interviews sometimes revealed that the cohort member was hospitalized outside the study area. Every effort was made to identify discharge diagnosis for such events and, when applicable, review hospital charts. However, events in other institutions providing medical care (such as nursing homes, rehabilitation hospitals, long-term care facilities, and psychiatric hospitals) were not investigated. Final MI classification was determined by Mortality and Morbidity Classification Committee reviews or a computer algorithm when the reviews were not required. After excluding 1 patient who was neither European American nor African American, 642 patients with incident MI (471 European American and 171 African American) were identified. In both suburban Minneapolis (129 patients) and Washington County (181 patients), the participants were exclusively European American. In Jackson (154 patients), participants were exclusively African American. In Forsyth County, both European American (161) and African American (17) patients were included. Of these 642 patients, 129 (82 European American and 47 African American) died subsequently during the follow-up. A total of $76.8 \%$ of the deaths in European American patients (63) and 80.9\% of the deaths in African American patients (38) occurred $\geq 29$ days after the hospital admission. The follow-up period was from the time of admission to death or to December 31, 1998, whichever occurred first. The median follow-up time was 3.7 and 3.3 years for European American patients and African American patients, respectively.

The baseline information, including age, sex, race, body mass index (BMI), smoking status, cigarette years of smoking, drinking status, alcohol drinking, low-density lipoprotein (LDL) cholesterol level, high-density lipoprotein (HDL) cholesterol level, total cholesterol level, sports index, hypertension, diabetes mellitus, education level, and household income, was collected by certified interviewers through home interviews and clinical examinations. Race was defined as European American (white) or African American (black) according to the participant's self-report. Smoking status was defined as current, former, never, and unknown. Cigarette years of smoking was defined as the average number of cigarettes per day times the number of years smoked. Drinking status was defined as current, former, never, and unknown on alcohol use. Alcohol drinking was defined as the usual number of grams per week of alcohol drinking. Sports index was calculated on the basis of the frequency and intensity of sports-related activities during leisure time. Hypertension was defined as systolic blood pressure $\geq 160 \mathrm{~mm} \mathrm{Hg}$, diastolic blood pressure $\geq 95 \mathrm{~mm} \mathrm{Hg}$, or taking medications for high blood pressure. Diabetes mellitus was defined as fasting blood glucose level $\geq 140 \mathrm{mg} / \mathrm{dL}$, non-fasting blood glucose level $\geq 200 \mathrm{mg} / \mathrm{dL}$, self-reported diabetes mellitus, or taking medications for diabetes mellitus. Methods have been described previously for the measurement of $\mathrm{BMI},{ }^{8}$ cholesterol level, ${ }^{8}$ sports index, ${ }^{9}$ and systolic and diastolic blood pressure. $^{8}$ Education level was defined as: 1, grade school or 0 years education; 2 , high school, but no degree; 3 , high school graduate; 4 , vocational school; 5 , college; 6 , graduate school or professional school. Household income, income from all sources, was defined as: $1,<\$ 5000 ; 2, \$ 5000$ to $\$ 7999 ; 3, \$ 8000$ to $\$ 11,999 ; 4, \$ 12,000$ to $\$ 15,999 ; 5$, $\$ 16,000$ to $\$ 24,999 ; 6, \$ 25,000$ to $\$ 34,999 ; 7, \$ 35,000$ to $\$ 49,999 ; 8, \geq \$ 50,000$.

Several characteristics of MI events were collected from hospital charts. Presence of shock or congestive heart failure during MI events was identified. An initial systolic blood pressure $<100 \mathrm{~mm} \mathrm{Hg}$ was defined as abnormal. Presence of Qwave on initial electrocardiogram was defined according to the Minnesota Code. ${ }^{10}$ Abnormal enzyme levels were defined as any creatine kinase-myocardial band isoenzyme level present or twice the upper limit of normal during the first 4 days of MI events. In addition, time from symptoms to arrival at hospital was gathered from hospital charts. Information on the use of procedures such as coronary angioplasty, coronary bypass grafting, and other cardiac procedures (International Classification of Diseases-9th revision codes 36.0, 36.1, or 36.2) after the admission was also attained from hospital charts.

Selected characteristics of patients with MI, by race, were examined. For European American and African American patients, unadjusted 3-year case fatality rates were calculated with the Kaplan-Meier method. Cox proportional hazard models were used to further explore the racial disparity. The assumption of proportionality was satisfied.

In the first model, the age- and sex-adjusted racial disparity in mortality rate after MI was investigated. Other factors were then added to the model separately to assess their contributions to the racial disparity. Because the time of performance of cardiac procedures was available, the use of cardiac procedures was assessed as a time-dependent variable in all analyses. For simplicity, these factors were also grouped into 4 sets: vascular risk factors, including BMI, smoking status, cigarette years of smoking, drinking status, alcohol drinking, LDL level, HDL level, total cholesterol level, hypertension, diabetes mellitus, and sports index; socioeconomic position, including education and income; severity of MI, including presence of shock, congestive heart failure during MI events, initial systolic blood pressure $<100 \mathrm{~mm} \mathrm{Hg}$, Q-wave, and abnormal enzyme levels; and treatment, including time from symptoms to arrival at hospital and use of cardiac pro- 
Table I. Distributions of characteristics by race in the 642 hospitalized patients with MI from the ARIC study, 1987-1998

\begin{tabular}{|c|c|c|c|}
\hline & $\begin{array}{l}\text { European } \\
\text { Americans } \\
(n=471)\end{array}$ & $\begin{array}{c}\text { African } \\
\text { Americans } \\
(n=171)\end{array}$ & $\boldsymbol{P}$ \\
\hline Age at time of diagnosis of incident $\mathrm{Ml}(\mathrm{y})$ & $62.2 \pm 6.2$ & $61.3 \pm 5.9$ & .09 \\
\hline Male (\%) & 68.8 & 48.5 & .001 \\
\hline BMI at baseline, $\left(\mathrm{kg} / \mathrm{m}^{2}\right)$ & $27.8 \pm 4.9$ & $29.6 \pm 5.8$ & .001 \\
\hline Cigarette years of smoking at baseline & $555.0 \pm 532.1$ & $345.2 \pm 437.7$ & .001 \\
\hline Alcohol drinking (g/week) & $40.6 \pm 84.5$ & $29.0 \pm 71.5$ & .12 \\
\hline LDL at baseline (mg/dL) & $151.7 \pm 36.6$ & $155.2 \pm 50.2$ & .35 \\
\hline $\mathrm{HDL}$ at baseline (mg/dL) & $42.0 \pm 12.3$ & $47.8 \pm 15.7$ & .001 \\
\hline Total cholesterol at baseline (mg/dL) & $226.0 \pm 39.9$ & $231.0 \pm 53.5$ & .21 \\
\hline Sport index at baseline & $2.4 \pm 0.8$ & $2.1 \pm 0.7$ & .001 \\
\hline Hypertension at baseline (\%) & 37.0 & 67.2 & .001 \\
\hline Diabetes at baseline (\%) & 19.6 & 31.7 & .001 \\
\hline Education ( $\geq$ high school) at baseline (\%) & 70.2 & 46.8 & .001 \\
\hline Income (>16k/year) at baseline (\%) & 81.4 & 37.1 & .001 \\
\hline Shock during $\mathrm{Ml}$ events (\%) & 2.6 & 2.3 & .88 \\
\hline Congestive heart failure during $\mathrm{Ml}$ events (\%) & 23.6 & 28.1 & .24 \\
\hline Initial systolic blood pressure $<100 \mathrm{~mm} \mathrm{Hg}(\%)$ & 6.4 & 3.1 & .12 \\
\hline$Q$ wave $(\%)$ & 12.6 & 14.2 & .27 \\
\hline Abnormal enzyme levels (\%) & 86.4 & 78.8 & .02 \\
\hline Time from symptoms to arrival at hospital ( $<6$ hour) (\%) & 57.0 & 52.7 & .27 \\
\hline Cardiac procedures after occurrences of $\mathrm{Ml}(\%)$ & 57.1 & 21.6 & .001 \\
\hline
\end{tabular}

Values presented as mean $\pm \mathrm{SD}$ or percent.

cedures. The extent to which each set of factors contributes to the racial disparity in mortality rate after MI was assessed. Finally, the model, including all factors aforementioned, was used to explore any residual racial disparity.

These analyses were also repeated, limiting the follow-up to the period from the 29th day since the admission to death or December 31, 1998. The previous ARIC report indicated that among patients with acute coronary heart disease who died within 28 days after MI events, more than half died before a hospital admission, and African American patients were more likely to die out of the hospital than European American patients. ${ }^{7}$ Because out-of-hospital deaths may be less likely to occur $\geq 28$ days after MI events, the racial difference in mortality rate during the period from the 29th day since the admission to death or December 31, 1998, may less likely be biased by out-of-hospital deaths.

\section{Results}

The distribution of the baseline characteristics of 642 patients who were hospitalized for MI was calculated by race (Table I). The proportion of men was less in African American patients than in European American patients (the same difference found in the entire ARIC cohort). BMI in African American patients was moderately higher than in European American patients. African American patients had smoked less than European American patients. The sports index in African American patients was lower than in European American patients. African America patients were substantially more likely to have hypertension and diabetes mellitus. Only $46.8 \%$ of African American patients had high school or higher education level, whereas 72.0\% of European American patients had high school or higher education level. The minority of African American patients were in families with income $>\$ 16,000$ (37.1\%), whereas most European American patients were in families with income $>\$ 16,000$ (81.4\%). There was no apparent difference in severity of MI or time from symptoms to arrival at hospital between African American and European American patients. However, cardiac procedures were much less frequently performed in African American patients than European American patients.

The unadjusted 3-year case fatalities showed a substantially worse outcome after MI for African American patients (21\%) compared with European American patients (14\%). After adjusting for age and sex, the relative hazard $(\mathrm{RH})$ of death after MI was 1.80 (95\% CI, 1.24- 2.61) for African American patients compared with European American patients (Table II). With further adjustment for each of the other factors individually, only sports index, hypertension, diabetes mellitus, income, and use of cardiac procedures attenuated the racial disparity to varying degrees. The largest attenuation was observed in the model with income, in which the RH for African American patients compared with European American patients decreased to 1.31 (95\% CI, 0.83-2.09). After adjusting for the 4 sets of factors, the RH for African American patients compared to European American patients decreased to 1.29 (95\% CI, 0.83-2.00), 1.31 (95\% CI, 0.83-2.09), 1.60 (95\% CI, 
Table II. Relative hazards of death after MI for 642 hospitalized patients with MI (129 deaths) in the ARIC Study, 1987-1998

Race (African Americans vs European Americans)

Relative hazard $(95 \% \mathrm{Cl})$

Demographics* adjusted

Demographics and BMI adjusted

Demographics, smoking status, and cigarette years of smoking adjusted

Demographics, drinking status, and alcohol drinking adjusted

Demographics, LDL, HDL, and total cholesterol adjusted

Demographics and sports index adjusted

Demographics and hypertension adjusted

Demographics and diabetes adjusted

Demographics and education adjusted

Demographics and income adjusted

Demographics, shock, and congestive heart failure adjusted

Demographics and initial systolic blood pressure $<100 \mathrm{~mm} \mathrm{Hg}$ adjusted

Demographics and $Q$ wave adjusted

Demographics and abnormal enzyme levels adjusted

Demographics and time from symptoms to arrival at hospital adjusted

Demographics and cardiac procedures adjusted

*Age and sex.

Table III. Relative hazards of death after MI for 642 hospitalized patients with MI (entire follow-up) and for 608 hospitalized patients with MI (limited follow-up: 448 European Americans and 160 African Americans) who survived at least 28 days after the admission in the ARIC Study*
$1.80(1.24-2.61)$

$1.84(1.26-2.67)$

$1.80(1.23-2.64)$

$1.73(1.18-2.55)$

$1.96(1.34-2.87)$

$1.55(1.06-2.25)$

$1.65(1.12-2.44)$

$1.63(1.12-2.38)$

$1.71(1.17-2.49)$

1.31 (0.83-2.09)

$1.70(1.17-2.48)$

$1.81(1.19-2.74)$

$1.83(1.26-2.66)$

$1.75(1.20-2.55)$

$1.74(1.20-2.54)$

$1.42(0.97-2.08)$

\begin{tabular}{lrr} 
& \multicolumn{1}{c}{ Relative hazard (95\% Cl) } \\
\cline { 2 - 2 } Race (African Americans vs European Americans) & Entire follow-up & Limited \\
follow-up \\
\hline Adjusted for demographics $\dagger$ & $1.80(1.24-2.61)$ & $1.90(1.25-2.89)$ \\
Adjusted for demographics and vascular risk factors & $1.29(0.83-2.00)$ & $1.33(0.80-2.20)$ \\
Adjusted for demographics and socioeconomic position & $1.31(0.83-2.09)$ & $1.43(0.85-2.38)$ \\
Adjusted for demographics and severity of MI & $1.60(1.05-2.45)$ & $1.72(1.12-2.66)$ \\
Adjusted for demographics and treatment & $1.36(0.92-2.00)$ & $1.40(0.91-2.16)$ \\
Adjusted for all above & $1.00(0.56-1.77)$ & $1.06(0.60-1.90)$ \\
\hline
\end{tabular}

*There were 129 (82 European Americans and 47 African Americans) deaths during entire follow-up and 101 deaths during limited follow-up ( 63 European Americans and 38 African Americans)

†Age and sex.

1.05-2.45), and 1.36 (95\% CI, 0.92-2.00) for vascular risk factors, socioeconomic position, severity of MI, and treatment, respectively (Table III). The largest reduction was observed in the model with vascular risk factors. In the final model with all the factors aformentioned, the RH for African American patients compared with European American patients was 1.00 (95\% CI, 0.56-1.77). When the follow-up was limited to the period from the 29th day since the admission to death or December 31, 1998, the pattern remained.

To examine the interactions of race with sex, hypertension, and diabetes mellitus, the fully adjusted analysis was used, stratified by these possible modifiers. The fully adjusted RHs for Africans American patients compared to European American patients were 0.84 (95\%
CI, 0.35-2.00), 1.35 (95\% CI, 0.59-3.09), 0.72 (95\% CI, 0.21-2.48), 0.96 (95\% CI, 0.47-1.97), 1.35 (95\% CI, $0.58-3.14)$, and 0.76 (95\% CI, 0.26-2.21) for women, men, patients without hypertension, patients with hypertension, patients without diabetes mellitus, and patients with diabetes mellitus, respectively. None of the interaction of race with sex, hypertension, and diabetes mellitus was statistically significant $(P>.05)$.

\section{Discussion}

In accordance with most previous studies, ${ }^{2-4}$ the unadjusted mortality rate after MI was worse in African American patients than European American patients in this study. However, there was no racial disparity after 
adjusting for vascular risk factors, socioeconomic position, severity of MI, and treatment.

Previous studies suggested that there was little disparity in short-term mortality rate after MI between African American patients and European American patients, ${ }^{2,3}$ even without adjustment for other factors. A previous analysis of the ARIC data from 1987 to 1993 showed that age-adjusted 28-day case fatality rates did not differ significantly by race among patients who were hospitalized for MI. ${ }^{7}$ However, more African American than European American patients with MI died before a hospital admission, and when out-of-hospital coronary heart disease deaths were included, the age-adjusted 28-day case fatality rates were higher in African American patients than in European American patients. Epidemiologic studies on racial differences in long-term mortality rate after MI are sparse. In 1 clinical trial, the unadjusted 1-year mortality rate of African American patients was significantly higher than that of European American patients (relative risk, 1.7; 99\% CI, 1.0-3.2). ${ }^{3}$ In another clinical trial, the unadjusted cumulative mortality rate at 48 months was 34\% for African American patients versus 24\% for European American patients $(P<.005) .^{2}$ As in observational studies, unadjusted relative risks were likely to be confounded in those trials, because race was not randomized. In this study, the long-term, unadjusted mortality rate fter hospitalization for MI was almost 2 times as great in African American patients as in European American patients. Out-of-hospital deaths are more likely to occur in patients with MI who died soon after the MI event. Because most deaths occur after the first 28 days in a long-term follow-up study, out-of-hospital deaths are less likely to contribute to racial differences in long-term mortality rates after hospitalization for MI Thus, although estimates of short-term racial differences in prognosis after MI may be affected by omission of these deaths, studies of long-term mortality rate (such as ours) are less likely to be affected by them.

As previously reported, ${ }^{2-4}$ in this study, some vascular risk factors, such as hypertension, diabetes mellitus, and obesity, were more prevalent in African American patients than European American patients. More African American patients had lower socioeconomic position. Although there was no apparent difference in severity of MI or time from symptoms to arrival at hospital between African American patients and European American patients, fewer African American patients had cardiac procedures. Adjusted analyses showed that vascular risk factors, socioeconomic position, and treatment contributed to most of the racial disparity in mortality rate after $\mathrm{MI}$.

The findings that hypertension and diabetes mellitus contributed to the racial disparity is consistent with previous work. In both the Thrombolysis in Myocardial Infarction phase II study and Multicenter Investiga- tion of the Limitation of Infarct Size Study, hypertension and diabetes mellitus contributed importantly to the racial disparity in mortality after MI. ${ }^{2,3}$ The underlying reasons for higher prevalence of those conditions in African American patients were not assessed in this study.

In this study, income was the most significant contributor to the racial disparity. This is not unexpected because income is likely to be related to measured and unmeasured vascular risk factors and to severity and treatment factors potentially related to mortality after MI. ${ }^{11}$ Education not being as strong a predictor is consistent with work documenting lower income returns to education in African American patients than in European American patients. ${ }^{12}$ Nonetheless, the differences in socioeconomic position between African American patients and European American patients may not be fully captured by the income and education variables used. $^{13}$

The use of cardiac procedures was an important contributor to the racial disparity in this study. A historical cohort study of acute MI in New Jersey found that African American patients had approximately 30\% lower odds of undergoing cardiac catheterization and revascularization within 90 days than European American patients after adjustment. ${ }^{14}$ In a study of 1987 Medicare beneficiaries with a recent acute myocardial infarction in the United States, the adjusted odds ratio for the use of angiography within 90 days, comparing African American patients with non-African American patients, ranged from 0.41 to $0.94 .{ }^{15} \mathrm{~A}$ recent study suggested that the racial bias of physicians affected their decision to refer patients with chest pain for cardiac catheterization. ${ }^{16}$

Long-term assessment of mortality after first MI in the well-defined communities is the strength of this study. An important limitation is that, for the most part, African American patients and European American patients resided in different states. In a subgroup analysis (restricted to Forsyth County), although the racial disparity was not statistically significant because of the small sample size, the results still showed a similar pattern (data not shown). Nevertheless, the different regions from which African American and European American patients were sampled limits our ability to draw inferences about racial differences in the United States generally.

In summary, this study suggested that vascular risk factors, socioeconomic position, and treatment are major contributors to the long-term racial disparity in mortality rate after MI.

We thank the staff and participants in the ARIC Study for their important contributions. 


\section{References}

1. Castaner A, Simmons BE, Mar M, et al. Myocardial infarction among black patients: poor prognosis after hospital discharge. Ann Intern Med 1988;109:33-5.

2. Tofler GH, Stone PH, Muller JE, et al. Effects of gender and race on prognosis after myocardial infarction: adverse prognosis for women, particularly black women. J Am Coll Cardiol 1987;9: 473-82.

3. Taylor HA, Chaitman BR, Rogers WJ, et al. Race and prognosis after myocardial infarction: results of the Thrombolysis in Myocardial Infarction (TIMI) phase II trial. Circulation 1993;88:1484-94.

4. Nakamura $Y$, Moss AJ, Brown MW, et al. Ethnicity and long-term outcome after an acute coronary event. Multicenter Myocardial Ischemia Research Group. Am Heart J 1999;138:500-6.

5. Peterson ED, Shaw LK, Delong ER, et al. Racial variation in the use of coronary-revascularization procedures: are the differences real? Do they matter? N Engl J Med 1997;336:480-6.

6. Jha AK, Shlipak MG, Hosmer W, et al. Racial differences in mortality among men hospitalized in the Veterans Affairs health care system. JAMA 2001;285:297-303.

7. White $A D$, Rosamond WD, Chambless LE, et al. Sex and race differences in short-term prognosis after acute coronary heart disease events: the Atherosclerosis Risk In Communities (ARIC) study. Am Heart J 1999;138:540-8.

8. National Heart, Lung, and Blood Institute. Atherosclerosis Risk in Communities (ARIC) Study manual of operations. Version $1.0 \mathrm{ed}$.
Chapel Hill, NC: ARIC Coordinating Center, School of Public Health, University of North Carolina; 1987.

9. Baecke JA, Burema J, Friiters JE. A short questionnaire for the measurement of habitual physical activity in epidemiological studies. Am J Clin Nutr 1982;36:936-42.

10. Kors JA, Crow RS, Hannan PJ, et al. Comparison of computerassigned Minnesota Codes with the visual standard method for new coronary heart disease events. Am J Epidemiol 2000;151: 790-7.

11. Kaplan GA, Keil JE. Socioeconomic factors and cardiovascular disease: a review of the literature. Circulation 1993;88:1973-98.

12. Cooper R, David R. The biological concept of race and its application to public health and epidemiology. J Health Polit Policy Law 1986;11:97-116.

13. Kaufman JS, Cooper RS, McGee DL. Socioeconomic status and health in blacks and whites: the problem of residual confounding and the resiliency of race. Epidemiology 1997;8:621-8.

14. Gregory PM, Rhoads GG, Wilson AC, et al. Impact of availability of hospital-based invasive cardiac services on racial differences in the use of these services. Am Heart J 1999;138:507-17.

15. Gatsonis CA, Epstein AM, Newhouse JP, et al. Variations in the utilization of coronary angiography for elderly patients with an acute myocardial infarction. An analysis using hierarchical logistic regression. Med Care 1995;33:625-42.

16. Schulman KA, Berlin JA, Harless W, et al. The effect of race and sex on physicians' recommendations for cardiac catheterization. N Engl J Med 1999;340:618-26. 